\title{
LECTORYN EN LA COMPRENSIÓN LITERAL DE ESTUDIANTES DE SECUNDARIA
}

\section{Lectoryn in the literal level of reading comprehension of high school students}

\begin{abstract}
Yngrid Marlene Rosadio Vega-Universidad César Vallejo*, Doris Fuster-Guillé-Universidad Nacional Mayor de San Marcos**, Ronald M. Hernández-Universidad San Ignacio de Loyola***, Pedro Félix Novoa Castillo-Universidad Nacional Mayor de San Marcos****, Eric Gálvez-Suárez-Universidad Nacional Mayor de San Marcos*****, Dante Aguinaga-Villegas-Universidad Nacional Mayor de San Marcos******
\end{abstract}

\begin{abstract}
Resumen
Uno de los problemas más críticos y aún irresueltos es la baja comprensión de textos en los escolares peruanos. Problemática en estado crítico corroborado por pruebas internacionales como las PISA (2015, 2018) y estudios diversos de investigadores sensibilizados por esta preocupante situación. El presente estudio investigativo buscó establecer la influencia del módulo "Lectoryn" en la comprensión literal de textos en estudiantes del primer año de Educación Básica Regular. Para lograr este objetivo, se utilizó un enfoque cuantitativo y se adoptó un diseño cuasi experimental. Como instrumento de recojo de información se empleó pruebas (pre test y post test) a una muestra intacta de 51 estudiantes (grupo de control, $n=24 ; y$ grupo experimental, $n=27$ ). Los resultados establecieron que en el pre test ambos grupos se ubicaban en Inicio y que luego del desarrollo del módulo "Lectoryn" el grupo Experimental ascendió al nivel de logro previsto, mientas que el grupo de Control no. Para la contrastación de hipótesis se empleó la prueba U the Mann-Whitney que demostró estadísticamente que en el pre test no había diferencias significativas entre ambos grupos, pero que en el post test, sí se manifestaban diferencias. Comprobándose la hipótesis de que el desarrollo del módulo "Lectoryn" mejora el nivel de comprensión literal.
\end{abstract}

Palabras claves: Comprensión literal, técnica de lectura, estrategia de lectura, programa educativo.

\begin{abstract}
One of the most critical and still unsolved problems is the low reading comprehension of texts in Peruvian high school students. This critical problem has been corroborated by international tests such as PISA $(2015,2018)$ and a variety of studies by researchers who have become aware of this worrying situation. The present research study aims to establish whether the Lectoryn module had any influence on literal reading comprehension of texts in students in the first year of Basic Regular Education. The study used a quantitative approach and a quasi-experimental design. nAs data collection instruments, tests (pre-test and post test) were used on an intact sample of 51 students (control group, $n=24$; and experimental group, $n=27$ ). The results showed that in the pre-test both groups were at the Initial level and, after the implementation of the Lectoryn module, the experimental group improved to the level of expected achievement, whereas the control group did not. For contrasting the hypothesis, the Mann-Whitney U test was used, which showed, statistically, that there were no significant differences in the pre-test between the two groups, whereas, in the post test, there were evident differences. Consequently, the hypothesis that the implementation of the Lectoryn module improves the literal level of reading comprehension has been proven.
\end{abstract}

Keywords: Literal reading comprehension, reading technique, reading strategy, educational program.

\footnotetext{
* Educadora. https://orcid.org/0000-0002-9417-8357

** Educadora. https://orcid.org/0000-0002-7889-2243

*** Psicólogo.rhernandezv@usil.edu.pe. https://orcid.org/0000-0003-1263-2454

**** Educador. https://orcid.org/0000-0003-2186-7458

***** Educador. https://orcid.org/0000-0002-7642-5019

******* Educador. https://orcid.org/0000-0001-5166-0509
} 


\section{INTRODUCCIÓN}

Leer y tener una buena comprensión es una permanente preocupación tanto para la educación como para la sociedad en general. Hasta la actualidad aún no se ha logrado resolver, incluso, muchos estudios establecen preocupantes situaciones de falta de comprensión sobre todo en el nivel escolar(Llamazares, Cortés y Sánchez (2015). Es por ello que hay un importante intento de aminorar sus consecuencias y proponer alternativas de solución desde lo pedagógico como técnicas, programas y estrategias. En el Perú, se ha observado que los escolares leen poco y lo poco que leen no lo comprenden a cabalidad. En la gran mayoría no sobrepasan lo literal y se manifiesta una preocupante falta de niveles de inferencia y crítico valorativo de lo leído. Es así que la educación debe propugnar un lector ideal que logre no solo leer de manera textual, sino que entienda la información entre líneas, que deduzca y tenga una actitud crítica. Para ello debe existir una condición activa entre el lector y el texto, lo cual lamentablemente está ausente en la mayoría de los estudiantes peruanos (Gordillo y Restrepo, 2012). Esta incapacidad para no comprender de manera integral los textos repercute en su pensamiento lógico, ya que no logran tampoco resolver problemas matemáticos ni de otros cursos que requieran deducción, inferencia y valoración, provocando inseguridad, bajas calificaciones, y una endeble autoestima, al sentirse en desventaja con relación a los que sí comprenden bien los textos, las indicaciones y las formulación de preguntas (Acharandi, 2009, p.4) configurando a los llamados "analfabetos funcionales". Personas que sabiendo leer no leen bien.

Es por todo lo expuesto que la comprensión de textos se constituya como una de las prioridades de los centros de enseñanzas de todos los niveles, pero en especial en los niveles escolares. Blythe y Perkins citado en Cifuente, (2014, p.74), manifiestan que al lograr una buena comprensión se posibilita el entrenamiento de un gran grupo de actividades cognitivas, donde el pensamiento se enfoca en un solo tema, se establece relaciones, ejemplifica, aplica, encuentra evidencias, generaliza y logra una representación nueva de cualquier tema. Y precisamente para lograr todo esto, el estudiante debe alcanzar un entendimiento cabal de lo leído.
Solé (1992) entiende a la lectura como un hábito complejo donde se tiene que partir de un propósito específico, establecer requerimientos simultáneos, decodificar con holgura y rapidez e interactuar con el texto leído, contribuyendo para su interpretación con sus conocimientos previos y propias interpretaciones. Asimismo, con estos ejercicios se consigue a su vez, predecir e inferir información que se desprenda del texto leído. De ahí la complejidad de leer un texto, ya que activa procesamientos cognitivos y lingüísticos múltiples en la mente del lector referidos a lo semántico, sintáctico (Hoyos y Gallego, 2017).

Según Achaerandio (2009) la actividad lectora constituye un ejercicio constructivo, interactivo y estratégico, porque quien lee va construyendo con su esquema mental una nueva configuración, más rica y variada gracias a la lectura, basada en conocimientos previos y al nivel de experimentación e interpretación.

La comprensión de textos presenta diversos niveles que Achaerandio $(2009$, p. 3) caracteriza así: el "Microproceso" que consiste en el primer nivel donde se realiza la descodificación y codificación de un idioma; aquí se presenta el nivel literal de compresión, donde el sujeto identifica, descifra letras, fonemas, oraciones, palabras y su correspondiente descomposición en niveles más bajos aún; también se presenta la creación de nuevas palabras, de reconfigurar su significado, su pronunciación, entre otros. El autor manifiesta que este nivel básico es muchas veces el techo de muchos alfabetizados, que no pueden salir de este y migrar a otro superior, configurando problemas graves de comprensión, ya que, en vez de ejercer una lectura comprensiva, prefirieron una del tipo mecánica. Con relación a los significados comprensivos de las palabras, Montealegre, 2004, manifiesta que los significados lexicales se encuentran dentro del bagaje histórico y social, y se escapa del alcance del informante, así como continuan con su estado de inmutabilidad a lo largo de su desarrollo. Cabe anotar, que las palabras al ser la esencia de lo simbólico es el motivador primordial para que el hombre pase de un estadio de pensamiento meramente sensorial a otro de raciocinio complejo.

El macroproceso es según Achaerandio (2009, pp.4-5) el nivel más complejo que logra un lector que 
ha comprendido bien un texto. Este segundo estadio permite realizar múltiples funciones comprensivas como captar el objetivo primordial del texto, saber la finalidad de la lectura, encontrarle un sentido. En este nivel se aprovecha con mayor énfasis los saberes previos, los conflictos cognitivos y su relación más eficientes con los nuevos aprendizajes. Asimismo, en este nivel se identifican las ideas principales, las secundarias, la cohesión y coherencia del contenido que contribuyan a una esquematización mental. También se realiza ejercicios mentales de inferencia y deducción, así como sus correspondientes comprobaciones y contrastaciones con lo que ya sabía o conocía sobre lo leído (Hoyos y Gallego, 2017). En este nivel se logra pasar de una comprensión textual a una inferencial haciendo uso de todas sus potencialidades mentales, y con el ejercicio eficaz de la detección de causalidades y otras relaciones entre ideas. Y, por último, se llega a la reflexión metacognitiva de comprender que uno está comprendiendo. Esto es imprescindible para la regulación efectiva del aprendizaje. Es así que, en este segundo nivel, la lectura puede llegar a una interesante sofisticación, donde el lector adquiere el protagonismo de su lectura, logrando mejor motivación, sentido de responsabilidad y el ejercicio de una actitud asertiva como sujeto activo, que reflexiona y critica. $\mathrm{Y}$ que precisamente por ello, se constituye como un estudiante con más autonomía y responsabilidad en su propio aprendizaje (Ramírez, Rossel \& Nazar 2015).

Dentro de los macroprocesos se toman por consiguiente "macrorreglas" al leer. Se toma en cuenta en ellas, la posibilidad en primera instancia de suprimir contenido irrelevante, impertinente que se identifica como una reiteración innecesaria; en segundo lugar, se consigue generalizar conceptos, importante procesamiento lector que permite consolidar conceptos, haciendo construcciones teóricas que sintetizan, jerarquizan y relacionan ideas implícitas. Y, por último, también encontramos a la integración. Esta última relacionada a la posibilidad de realizar resúmenes que agregan fragmentos relevantes del contenido de una lectura. Se concluye que los macroprocesos constituyen un nivel superior en el aprendizaje. Ya que el lector, sobrepasa el nivel textual y presentan facilidad para internalizar información nueva o reacomodada en sus estructuras mentales, considerando a quienes llegan a estos macroprocesos "buenos lectores". Ya que, según Munayco (2018), en este nivel se llega a un gran grado de interacción del texto con los esquemas cognitivos, donde estos se vuelven más dinámicos en la representación de una comprensión cada vez más eficiente, ágil y significativa.

En la misma sintonía, el Ministerio de Educación de Guatemala (MINEDUC) concibe la comprensión de textos como un procesamiento mediante el cual se llega a encontrar un sentido global de lo leído, encontrando un significado entre lo que se sabe y los nuevos conocimientos. Solé (1996, p.44) señala que esta significatividad se da cuando los esquemas cognitivos se enfrentan con la nueva información y obligan a estos a reconfigurarse. Este proceso es vital para que se vayan obteniendo más significados de mayor relevancia y significancia para los lectores. Para lograr ello, se necesita que se demuestre habilidades específicas (Quiñónez, 2012). Esto último guarda estrecha relación con lo sostenido por Solé, cuando puntualiza que, para lograr la comprensión y entendimiento de una lectura, es importante que se activen los saberes previos y se procure vincularlos con los nuevos conocimientos. La dificultad con la comprensión, reside en gran parte, que debido a que los estudiantes no cuentan con el suficiente conocimiento previo, el anclaje con los nuevos no se llega a dar. Debido a ello, se observa que el léxico es exiguo, y que por ello, ni siquiera sobrepasa una mera comprensión textual de lo que intentan leer y comprender.

Cassany (2001) menciona que la comprensión de un texto es un procesamiento integral, compuesto por componentes más básicos denominados micro habilidades (p.193). Estas exigen un relativo entrenamiento para incrementar el área de visión, la capacidad de discriminar ideas, conceptos. Es decir, comprender lo más significante. Otra, es el desarrollo de la memoria a corto plazo al igual que las habilidades de poder observar y anticipar información, sucesos y consecuencias con relación a otras ideas, personajes o sucesos. Asimismo, se debe incentivar una lectura veloz que a la par logre una comprensión también rápida. Una lectura inferencial, en el empleo de información extraída del propio texto, pero también la obtenida del contexto. Identificar las ideas más relevantes y las que siguen en jerarquía de importancia. Otra micro 
habilidad es tener la conciencia metacognitiva de la lectura e ir autorregulando el proceso de lectura, corrigiendo errores en el mismo proceso.

Novoa $(2018 ; 2019)$ enfatiza que la comprensión debe ser integral en el sentido de conseguir que el lector logre un pleno entendimiento en lo textual, inferencial y crítico valorativo. Por su parte, el Minedu (2007, p.16) apunta que la comprensión implica una interpretación. Es decir, se necesita asignarle un sentido a lo que se viene leyendo. Y que para que ese procesamiento sea eficaz, se debe ir avanzando de manera progresiva. Es así, que adquiere sentido la progresiva complejidad en los textos leídos, desde los textos con puras imágenes, los que van agregando textos a las imágenes y los puramente textuales que a su vez se deben ir complejizando de lo más simple a lo más exigentes tanto en lo formal como en el contenido. Muñoz y Ocaña (2017) indican que la habilidad de comprender un texto se puede detectar cuando se observa que un lector maneja un gran repertorio de palabras, que las comprende, usa y que es capaz de encontrar una determinada macroestructura de una lectura, que es capaz de reconocer las ideas principales como las inferenciales: en el ámbito lexical, el de causalidad, la comparación, la especificación, la inclusión y las diversas macroestructuras.

La comprensión en el nivel textual o literal se expresa cuando el estudiante recupera una información tal cual como la ha leído en el texto (MINEDUC, 2012). Pero se debe subrayar, que este tipo de comprensión es muy básica, y que, si solo los alumnos logran conseguir este nivel, los esfuerzos en el aula deberían enfocarse en desarrollar técnicas o metodologías idóneas para transitar a niveles más complejo de comprensión. Strang, 1965 citado por Gordillo y Flórez (2009, p. 97) y Smith (1989) coinciden en establecer que el nivel literal de la comprensión es donde los lectores pueden reconocer frases, palabras, sucesos o ideas expuestas explícitamente en el texto. Y que su logro implica una mínima actividad cognoscitiva por parte del lector. Para el Minedu (2007) la literalidad en la comprensión es la que se centra en la lectura tal cual. Se entiende bien lo que la lectura expresa y se recupera con precisión.

Para corroborar si un lector consigue la comprensión de textos en un nivel literal se debe tomar en cuenta el empleo de las siguientes habilidades (Minedu, 2007): Primero, la identificación de contenido, su localización, la detección de información relevante, la identificación de los tipos de texto (instructivos, argumentativos, narrativos, expositivos, etc.) y la reconstrucción secuencial de sucesos leídos. Segundo, el ejercicio de tomar decisiones de manera estratégica de acuerdo al tipo de texto, al momento de la lectura y al propósito de la lectura. Tercero y último, es la habilidad de reorganización del contenido leído, a través de la identificación de similitudes y discordancias entre la información obtenida en la lectura. Desde acciones, sucesos, personajes y otros datos hasta ideas explícitas o implícitas, con la capacidad de citar información tanto textual como parafraseado.

Por otro lado, se remarca que, si la comprensión es literal, esta implicará una perspectiva integral de todo el texto, así como la coherencia que siga un mismo eje vertebral durante toda la lectura, de tal manera que el lector sea capaz de relacionar todos los datos informativos dentro de un ámbito global (Guzmán, Fajardo, Duque 2015). En la comprensión literal, el lector logra identificar y recordar el contenido tal cual lo ha leído en el texto. En este nivel se explicitan dos procesamientos: uno léxico donde se decodifica o codifica de acuerdo al contexto y se accede a la significación empleando el vocabulario o lexicón mental; otro, llamado analítico, donde se mezclan las palabras con sus significados en una relación interpretativa e ideal (Vega, 2012, p.13). Para Fonseca, (2018,p.29) este tipo de comprensión textual, presupone captar lo que la lectura nos dice de manera directa y sin ambages, y a su vez, la capacidad de poder expresar con nuestras propias palabras lo que se ha entendido sin distorsionar la esencia de lo expresado en la lectura. Por todo ello, es muy importante que el nivel literal se dé de la mejor manera, ya que constituye la base para las otras comprensiones más complejas (Cuñachi y Leyva, 2018), ya que, sin estas, no se podrá lograr la comprensión global de todo el texto leído.

Es por todo lo anterior, que en la presente investigación se creyó conveniente la identificación del nivel en el que se hallaban los participantes. Para ello, se empleó el módulo Lectoryn que implica la innovación de estrategias y técnicas de lectura, para 
que sean un medio eficaz para los estudiantes en su necesidad de comprensión. Ya que, una vez logrado, el primer nivel literal, podrán ascender a otros niveles más complejos. Asimismo, el hecho de comprender cabalmente un texto, se expresaría en una mejoría evidente de su rendimiento escolar, tanto en el área de comunicación como en otras áreas.

El módulo "Lectoryn" se constituye, por tanto, como una alternativa novedosa para lograr que el primer nivel de comprensión se asegure. Ya que como se ha sustentado líneas arriba, logrando superar este primer estadio comprensivo, se puede avanzar a un nivel inferencial y crítico valorativo. El módulo, además, brinda estrategias tanto antes de la lectura, como durante la lectura y después de ella, para optimizar el entendimiento literal del texto leído. Potencializando sus habilidades en la lectura. Y asegurar así logros académicos que implicarán mejoras sustantivas en el Lenguaje y el pensamiento. Este módulo está compuesto por sesiones de aprendizajes de temáticas específicas, estrategias puntuales de acuerdo a objetivos previamente establecidos. Todo el módulo busca convertirse en un pilar de desarrollo en cada capacidad específica de los estudiantes. La finalidad de cada una de las actividades es potencializar el nivel de comprensión literal.

Tabla 1.

Módulo Lectoryn y sus técnicas de lectura (comprensión literal)

\begin{tabular}{ll}
\hline $\mathrm{N}^{\circ}$ Técnica & \multicolumn{1}{c}{ Capacidad } \\
\hline 1 Predicción & El lector es capaz de anticipar \\
& sucesos. Se aplica en el proceso, \\
& $\begin{array}{l}\text { tanto antes de la lectura como } \\
\text { después. }\end{array}$
\end{tabular}

2 Descripción El lector caracteriza elementos de lo leído que puede ser objetos, personas o sucesos a través de imaginarios mentales.

3 Comparación El lector establece diferencias y semejanzas entre personajes $\mathrm{o}$ sucesos.

4 Cadena secuencial El lector es capaz de proseguir una concatenación de sucesos.

5 Causalidad El lector tiene la capacidad de establecer causas y efectos.

6 Identificación temática El lector logra establecer el tema de toda la lectura, así como la de cada párrafo.
7 Entendimiento de palabras nuevas

8 Interrogatorio

9 Organizador visual de información.

10 El subrayado

11 El resumen

12 El esquema

\section{MÉTODO}

El estudio se circunscribe dentro del enfoque cuantitativo, siguiendo a Hernández Fernández y Baptista (2010, p. 6) es un tipo de investigación que estudia los fenómenos a través del empleo de mediciones estadísticas para establecer cierto patrón comportamental y/o llevar a la experiencia planteamientos teóricos. Y para ello suele plantearse preguntas relacionadas a determinar relaciones $\mathrm{o}$ interacciones de correlación, causalidad, o influencias entre variables de característica mensurable.La presente investigación optó por un diseño experimental, que constituye aquel que pretende averiguar las incidencia o influencia de una variable sobre otra.

La población del estudio, según Hernández y otros (2014, p. 174) como el universo focal que le interesa al investigador, se constituye como la totalidad de personas que guardan características observables en determinado tiempo y lugar. En específico, para esta investigación se abordó una población de 51estudiantes del Primer grado de una I.E de Educación Secundaria de Lima (secciones "B" y "C").

La técnica utilizada es concerniente a la prueba y el instrumento de recolección de datos desarrollada por los investigadores. Para esto se tuvo que establecer los componentes teóricos siguiendo los pasos de la operacionalización de variables. La dimensión, 
comprensión literal consta de un total de tres reactivos; la segunda, toma de decisiones estratégicas está compuesta de dos reactivos; mientras que la tercera, reorganiza la información, presenta también dos reactivos. Las preguntas están dispuestas a manera de aseveraciones de opción múltiples donde la opción correcta es una sola, otras son preguntas tipo verdaderofalso, y también interrogantes de respuesta abierta. La administración de este instrumento fue de manera colectiva, con una duración de entre cuarenta y cinco a sesenta minutos. La baremación y rangos se estableció con los rangos: Inicio $(0-10)$, proceso $(11-13)$, logro previsto (14-17), y logro destacado (18-20). Puntaje catalogado con referencias al Ministerio de Educación. Y tipificada como una Prueba mixta orientada solo al nivel literal. La validación se realizó por contenido a través de juicio de expertos, entendiendo la validez como la verificación de certificar que el instrumento mida lo que realmente pretenda medir (Hernández y otros, 2014). Y con relación a la confiabilidad del instrumento, entendida por la reiterada certeza de resultados en diversos contextos, se tomó la Prueba KR-20 al ser en esencia un instrumento cuyos ítems son politómicos. El resultado de la confiabilidad resultó .832 y .811 , considerados altos.

Tabla 2

Fiabilidad de los instrumentos

\begin{tabular}{lcc}
\hline Test & Kuder Richardson (KR 20 & Ítems \\
\hline Pre test & .823 & 13 \\
Pos test & .811 & 13 \\
\hline
\end{tabular}

Se utilizó el software estadístico SPSS para procesar toda la información recogida. Dicho procesamiento dio origen al gráfico Caja de bigotes cuya interpretación de los valores mínimos y máximos servirán para apreciar de manera visual el comportamiento numérico de las variables. Y para la contrastación de las hipótesis se corroboró con la Prueba U de Mann-Whitney, ya que es la prueba idónea para verificar si existe diferencias significativas entre muestras independientes. Asimismo, se verificó que, en el descarte de la normalidad, determinando la naturaleza no normal y por ello la elección de una prueba no paramétrica como lo es la U de MannWhitney.

\section{RESULTADOS}

En el pretest, el $46 \%$ de los participantes del Grupo Control se posicionan en Inicio; al tiempo que el 38\% se posiciona en Proceso; el 17\% se ubica en Logro previsto; y ninguno (0\%) pudo ubicarse en nivel máximo nivel, Logro destacado, de la comprensión Literal de textos. Por su parte, los participantes del Grupo Experimental consiguieron los siguientes resultados: el 48\% se posicionó en Inicio; el 48\%, en Proceso; el $4 \%$, en Logro previsto y el $0 \%$, en Logro destacado de la Comprensión literal de textos.

En el postest, los participantes del Grupo Control obtuvieron los siguientes resultados: el $42 \%$ se posicionó en Inicio; el 42\%, en Proceso; el 13\%, en Logro previsto y el $4 \%$, en Logro destacado de la Comprensión literal de textos. Mientras que los participantes del Grupo Experimental tuvieron: el 7\% en Inicio; el 7\%, en Proceso; el 81\%, en Logro previsto; y el $4 \%$, en Logro destacado de la Comprensión literal de textos.

Tabla 3

La influencia del módulo "Lectoryn" en la Comprensión literal de textos en estudiantes de secundaria

\begin{tabular}{lrrrr}
\hline & \multicolumn{2}{c}{ Grupo control (n:24) } & \multicolumn{2}{c}{ Grupo experimental (n:27) } \\
Indicador & Frecuencia & Porcentaje & Frecuencia & Porcentaje \\
\hline Pre test & & & & \\
En inicio & 11 & $46 \%$ & 13 & $48 \%$ \\
En proceso & 9 & $38 \%$ & 13 & $48 \%$ \\
Logro previsto & 4 & $17 \%$ & 1 & $4 \%$ \\
Logro destacado & 0 & $0 \%$ & 0 & $0 \%$ \\
Media & 10.8 & & 10.13 & \\
Desviación standar & 3.0 & & 2.4 & \\
Pos test & & & & \\
En inicio & 10 & $42 \%$ & 2 & $7 \%$ \\
En proceso & 10 & $42 \%$ & 2 & $7 \%$ \\
Logro previsto & 3 & $13 \%$ & 22 & $81 \%$ \\
Logro destcado & 1 & $4 \%$ & 1 & $4 \%$ \\
Media & 10.9 & & 14.7 & \\
Desviación standar & 3.0 & & 2.0 & \\
\hline
\end{tabular}


Se puede observar en la figura 1, que la Comprensión literal de textos, en el pretest, los participantes del Grupo Experimental presentan semejanza con los participantes del Grupo control; a diferencia que la Comprensión literal de textos, en el pos test, los participantes del Grupo experimental evidencian marcada ventaja sobre los participantes del Grupo control, ya que en el Grupo experimental se desarrolló el módulo "Lectoryn".

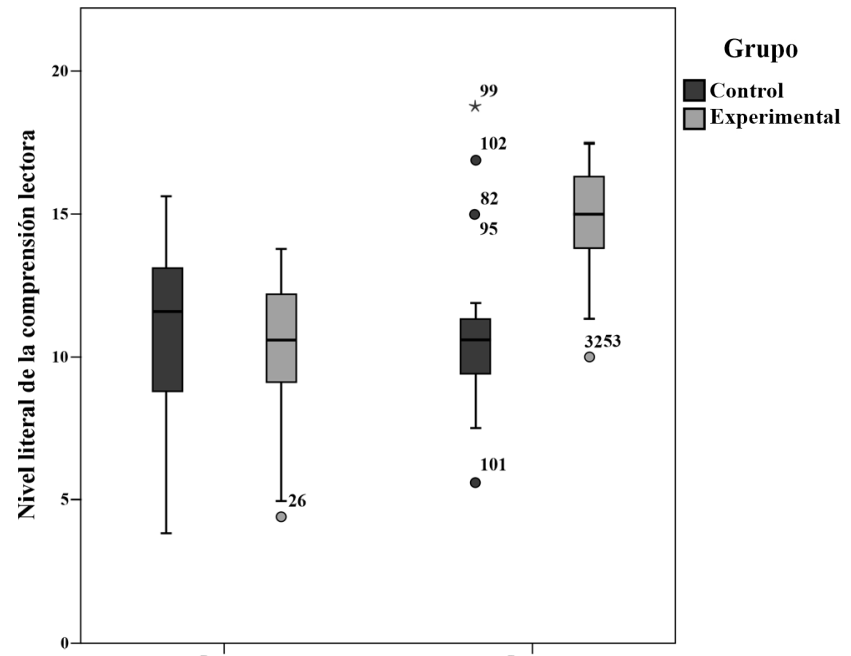

Figura 1: Módulo "Lectoryn" en la comprensión literal de estudiantes de Primer grado de una I.E de Educación Secundaria

En el pretest, los participantes del Grupo Control obtuvieron los siguientes resultados: el $75 \%$ se encontró en Inicio; el 21\%, en proceso; el 4\%, en logro previsto y el $0 \%$, en nivel logro destacado correspondientes a la dimensión Toma de decisiones estratégicas de la Comprensión literal de textos. Los participantes del Grupo Experimental tuvieron los siguientes resultados: el 89\% se ubicó en inicio; el $11 \%$, en proceso; el $0 \%$, en logro previsto y el $0 \%$, en nivel logro destacado correspondientes a la dimensión Toma de decisiones estratégicas en la Comprensión literal de textos.

En el postest, los participantes del Grupo Control tuvieron los siguientes resultados: el 50\% se posicionó en inicio; el 29\%, en proceso; el 17\%, en logro previsto; y el $4 \%$, en el nivel logro destacado. A diferencia de los participantes del Grupo Experimental, que obtuvieron: el $7 \%$ en inicio, el $22 \%$ en el nivel proceso, el $67 \%$ en logro previsto y el $4 \%$ está en nivel logro destacado, correspondiente a la Toma de decisiones estratégicas de la Comprensión literal de textos.

Tabla 4

Influencia del módulo "Lectoryn" en la toma de decisiones estratégicas respecto a la lectura la Comprensión literal de textos en los estudiantes de una I.E de Educación Secundaria Lima.

\begin{tabular}{lrrrr}
\hline \multirow{2}{*}{ Indicador } & \multicolumn{2}{c}{ Grupo control (n:24) } & \multicolumn{2}{c}{ Grupo experimental (n:27) } \\
& Frecuencia & Porcentaje & Frecuencia & Porcentaje \\
\hline Pretest & & & & \\
En inicio & 18 & $75 \%$ & 24 & $89 \%$ \\
En proceso & 5 & $21 \%$ & 3 & $11 \%$ \\
Logro previsto & 1 & $4 \%$ & 0 & $0 \%$ \\
Logro destacado & 0 & $0 \%$ & 0 & $0 \%$ \\
Media & 6.2 & & 4.8 & \\
Desviación standar & 4.3 & & 2.4 & \\
Postest & & & & \\
En inicio & 12 & $50 \%$ & 2 & $7 \%$ \\
En proceso & 7 & $29 \%$ & 6 & $22 \%$ \\
Logro previsto & 4 & $17 \%$ & 18 & $867 \%$ \\
Logro destcado & 1 & $4 \%$ & 1 & $4 \%$ \\
Media & 9.9 & & 14.1 & \\
Desviación standar & 4.8 & & 3.7 & \\
\hline
\end{tabular}

Como se evidencia en la tabla 5, no se presenta diferencias significativas en la Comprensión literal de textos entre el grupo de control y el grupo experimental durante el pretest. Pero, si se presenta diferencias significativas en la Comprensión literal de textos entre el grupo de control y el grupo experimental durante el postest, manifestando mayores resultados los participantes del grupo experimental. Esto demostrado estadísticamente por el valor de significancia durante el postest $\mathrm{p}=0.000$. Y al ser menor que $\alpha=0.05$, se resuelve rechazar la hipótesis nula. Comprobándose que la incidencia del módulo "Lectoryn" en la Comprensión literal de textos en los estudiantes de secundaria es significativa. Por consiguiente, se acepta la hipótesis alterna. 
Tabla 5.

Prueba $T$ de Student para comprobar medias de muestras independientes aplicada para hipótesis general

\begin{tabular}{lcc}
\hline Test & Indicador & Resultado \\
\hline \multirow{3}{*}{ Pretest } & $\mathrm{t}$ & $0.678^{*}$ \\
& $\mathrm{gl}$ & 49 \\
& Sig. asintónica (bilateral) & .501 \\
\cline { 2 - 3 } & & \\
\cline { 2 - 3 } Postest & U de Mann-Whitney & 105.500 \\
& 2 & -4.147 \\
& Sig. asintónica (bilateral) &, 000 \\
\hline
\end{tabular}

*/Varianzas iguales $(\mathrm{F}=1.795$, sig. $=0.186)$

\section{DISCUSIÓN}

El presente estudio se propuso establecer la incidencia del módulo "Lectoryn" en la Comprensión literal de textos de los estudiantes de una I.E de Educación Secundaria. Se consiguió corroborar las hipótesis de trabajo, ya que el desarrollo del módulo "Lectoryn" influenció de manera positiva en la comprensión literal de textos. Logro que se demostró al comprobarlo con la t-Student en el pre test y con la Prueba U de Mann Whitney para el postest. Donde el grupo de control llegó al $42 \%$ en Proceso; $13 \%$ en Logro previsto y $4 \%$ en logro destacado, mientras que el grupo experimental consiguió resultados superiores: en nivel de inicio $7 \%$, en proceso $7 \%$, logro previsto $81 \%$ y logro destacado $4 \%$. Resultados que confirman la significancia de 0,000; por consiguiente, se acepta la hipótesis general. Estos resultados coinciden con la investigación de Quispe (2013) donde se corrobora que el empleo de música instrumental presenta efectos positivos en la comprensión de textos de los estudiantes. En el mismo sentido, Morales (2012) en su investigación indica que la aplicación de un programa de lectura silenciosa incrementó, pero no de manera significativa la comprensión lectora. Estos resultados se explicarían por un diseño no tan idóneo del programa, que la lectura no haya provocado interés para los estudiantes, el nivel social y económico de los participantes, su ritmo personal de aprendizaje, un método poco efectivo, un vocabulario exiguo y hasta precario, y hasta participantes con serios problemas en la comprensión literal de los textos leídos. Al ser esto último cierto, se entendería que, al no llegar al primer nivel textual, no podrían haber llegado a otros niveles más complejos como el inferencial o criterial. Precisamente por ello, en este estudio se cuidó que estos factores no interfirieran. Se colige que podría haberse teorizado más la variable. Para que así, se optara por una estrategia de resolución más adecuada.

Por otro lado, el módulo "Lectoryn" incrementó los resultados en lo referido a identificación de información en los participantes del Grupo Experimental. El resultado del Grupo Control fue en inicio de $42 \%$, en proceso $21 \%$, logro previsto $33 \%$ y logro destacado de 4\%; mientras que para el Grupo Experimental: inicio con el $11 \%$, en proceso $15 \%$, logro previsto $56 \%$ y logro destacado de 19\%. Asimismo, se comprobó a través de la t-Student en el pretest y en el postest con la Prueba U de Mann- Whitney, donde se corroboró la significancia de 0,000, dando por consecuencia la aceptación de la hipótesis de trabajo. También el módulo "Lectoryn" incrementó la dimensión Toma de decisiones estratégicas con respecto a la lectura literal de la compresión de textos en los participantes del grupo experimental. Los resultados del grupo de control fueron del $50 \%$ de participantes ubicados en inicio; el $17 \%$ posicionados en proceso; el $17 \%$ en logro previsto y el $13 \%$ en logro destacado. Contrariamente, el grupo experimental resultó con resultados superiores: el $7 \%$ en el inicio, el $30 \%$ en proceso, el $26 \%$ en logro previsto y $37 \%$ en logro destacado. Esto demostrado por la Prueba U de Mann Whitney en el pre test y pos test, donde se demostró que había significatividad de 0,000, por consiguiente esto determinó la aceptación de la segunda hipótesis de la trabajo. Ambos hallazgos entran en contrastación con los realizados por Martínez (2011) demostró que las herramientas TIC devienen a potentes medios de apoyo y fortalecimiento en la comprensión de textos. Estrategia que dio muy buenos resultados al ser empleados en Colombia. Por otro lado, se puede hacer una comparación de resultados con lo hallado por Arbaiza, Orejuela y Sánchez (2011) donde se decidió emplear el programa PVC para la mejora del vocabulario y la comprensión de textos. Los hallazgos conseguidos posterior a la prueba t-Student dio un valor de $t=0,815$. Dato que es no significativo ( $p \geq$ $0,05)$, concluyendo en el correspondiente rechazo de la hipótesis general. Se infiera que en el estudio no funcionó como se había proyectado, porque se habría empleado todavía una modalidad un tanto tradicional, 
o no la cantidad de técnicas y estrategias necesarias, o también participantes que a lo mejor no habrían llegado a generar un conflicto cognitivo en las sesiones y su correspondiente aprendizaje significativos, o también se podría haber dado el caso de falta de un adecuado hábito lector, o participantes con dificultades de audición, de visión u otros.

Para finalizar, el módulo "Lectoryn" incrementó la dimensión Reorganizar la información de textos en los participantes del grupo experimental. El resultado del Grupo Control fue del 54\% en inicio, el $17 \%$ en proceso, $17 \%$ en logro previsto y el $13 \%$ en logro destacado. Por el contrario, en el Grupo Experimental los resultados fueron muy superiores: el $7 \%$ en inicio, el $30 \%$ en proceso, el $26 \%$ en logro previsto y el $37 \%$ en logro destacado. Esto se contrastó a través de la Prueba U de Mann Whitney para el pretest y la t-Student para el postest, donde se corrobora que se presenta significatividad de 0,000 , y por tanto se rechaza la hipótesis nula y se acepta la tercera hipótesis de trabajo. Así, se demuestra que el módulo "Lectoryn" planificado y utilizado en una I.E de Educación Secundaria resultó eficaz. Este hallazgo coincide con Quispe (2013) quien comprueba que el empleo de música instrumental evidenció incidencias favorables en los participantes y consiguió mejorar significativamente la comprensión de textos de los participantes en su investigación.

\section{REFERENCIAS}

Achaerandio, L. (2009). Lectura comprensiva. Guatemala: Universidad Rafael Landivar.

Arbaiza, C., Orejuela B. y Sánchez A. (2012) Eficacia del programa para la potenciación del vocabulario receptivo y el nivel de comprensión lectora en estudiantes de primer año de educación secundaria. Tesis de Maestría. Lima. Pontificia Universidad Católica del Perú.

Cassany, D. (2006). Tras las líneas. Sobre la lectura contemporánea. Barcelona: Anagrama.

Cegarra, S. (2012). Los métodos de investigación. ( $1^{\circ}$ ed.). México: Pearson Educación
Cifuente, J (2014). Enseñanza para la comprensión: opción para mejorar la educación. Revista Educación y Desarrollo Social, 9 (1), 70-81.

Cuñachi, G. y Leyva, G. (2018) Comprensión lectora y el aprendizaje en el área de Comunicación Integral en los estudiantes de Educación Básica Alternativa de las instituciones educativas del distrito de Chaclacayo UGEL 06 Ate- Vitarte. (Tesis para optar la licenciatura en Educación) Universidad Nacional de Educación Enrique Guzmán y Valle.

Fonseca, Y. (2018) Nivel de comprensión lectora de los estudiantes de quinto grado de primaria de la Institución Educativa $N^{\circ} 32385$ "Virgen De Fátima", Distrito de Llata, Provincia De Huamalíes, Región Huánuco. (Tesis para optar el título de licenciada en educación) Universidad Católica Los Ángeles Chimbote.

Fraca, L. (2003). Pedagogía Integradora en el aula. Los libros de El Nacional.Caracas, Venezuela: CEC.

Gordillo Alfonso, A. y, Restrepo Becerra, J. (2012) Comprensión lectora y concepciones de estudiantes universitarios sobre enunciados matemáticos. Zona Próxima (17), 2-23.

Gordillo, A. y Flórez, M. (2009). Los niveles de comprensión lectora: hacia una enunciación investigativa y reflexiva para mejorar la comprensión lectora en estudiantes universitarios. Revista Actualidades Pedagógicas, 53, 95-99.

Guzmán Torres, Leidy, Fajardo Martha, Duque Claudia (2015) Comprensión y Producción Textual Narrativa en Estudiantes de Educación Primaria. Revista Colombiana de Psicología, 24(1), 61-83.

Hernández Sampieri, R., Fernández Collado, C., \& Baptista Lucio, P. (2014). Metodología dela investigación (5ta. ed.). México: Interamericana.

Hoyos, A. \& Gallego, T. (2017). Desarrollo de habilidades de comprensión lectora en niños y niñas de la básica primaria. Revista Virtual Universidad Católica del Norte, 51, 23-45.

Llamazares Prieto, T., Cortés Fradejas, D., y Sánchez Rodríguez, S. (2015) Factores que influyen en el aprendizaje de la comprensión lectora y de la 
composición escrita: tres estudios realizados en aulas de Educación Infantil y Educación Primaria Universidad de Cádiz. Investigadores sobre la lectura, (3), 67-82.

Martínez Mejía, R. y Rodríguez Villanueva ,B . (2011). Estrategias de comprensión lectora mediadas por la TIC una alternativa para mejorar las capacidades lectoras en secundaria. Escenarios, 9 (2), 18-25.

MINEDUC. (2012). Guía Docente para la comprensión de lectura. Guatemala.

Ministerio de Educación. (2007). Guía de Estrategias Metacognitivas para Desarrollar la Comprensión Lectora. Niveles de la comprensión lectora, 103. Lima, Perú.

Montealegre, Rosalía (2004) La comprensión del texto: sentido y significado. Revista latinoamericana de Psicología, 36(2), 243-255

Morales de López, L. F. (2012). Incidencia del programa de lectura silenciosa en el nivel de comprensión lectora de las educandas de segundo básico de un colegio privado para señoritas ubicado en la ciudad de Guatemala. Facultad de Humanidades. Universidad Rafael Landívar. Guatemala.

Munayco Medina, Alfredo. (2018). Influencia de los organizadores gráficos en la comprensión lectora de textos expositivos y argumentativos.Comuni@ cción, 9(1), 05-13.

Muñoz-Muñoz, Á., \& Ocaña De Castro, M. (2017). Uso de estrategias metacognitivas para la comprensión textual. Cuadernos de Lingüística Hispánica, (29), 223-244.

Novoa Castillo, P. (2019) El Mapa mental armónico en la comprensión y producción de textos narrativos en estudiantes de pregrado de la UCV (Novoa Castillo, Pedro. Tesis de doctorado, Universidad César Vallejo). Lima, Perú.

Novoa Castillo, P. F., Cancino Verde, Francisca, R., Flores Sotelo, W. S., \& Nieto Gamboa, J. (2018).

Fecha de recepción: 3 de octubre, 2019

Fecha de aceptación: 29 de octubre, 2019
El Mapa Mental Armónico en la comprensión de textos narrativos en estudiantes universitarios. Propósitos y Representaciones, 6(2), 541-573. doi: 10.20511/pyr2018.v6n2.243.

Novoa Castillo, P. F., Verde, C., Francisca, R., Flores Sotelo \& W. S., Nieto Gamboa, J. (2018). The Harmonic Mind Map in the Comprehension of Narrative Texts in University Students. Journal of Educational Psychology-Propositos y Representaciones, 6(2), 575-606.

Novoa, P. et al. (2018). El Mapa Mental Armónico en la comprensión de textos narrativos en estudiantes universitarios. Propósitos y Representaciones, 6(2), 541-573.

Quispe Huacho, J. (2013). Aplicación de la música instrumental en la comprensión lectora de los estudiantes del 1er. grado de educación secundaria de la I.E.P. Juan Enrique Newman. (Tesis de Maestría en docencia y gestión educativa) Universidad César Vallejo. Lima, Perú.

Quiñónez, A. (2012). Uso de claves de contexto. Una estrategia para leer comprensivamente. Tercer grado del Nivel de Educación Primaria. ( $2^{\mathrm{a}}$ ed.) Guatemala: Dirección General de Evaluación e Investigación Educativa. Ministerio de Educación.

Ramírez Peña, Pamela, Rossel Ramirez, \& Nazar Carter, Gabriela. (2015). Comprensión lectora y metacognición: Análisis de las actividades de lectura en dos textos de estudio de la asignatura de Lenguaje y Comunicación de séptimo año básico. Estudios pedagógicos, 41(2), 213-231. doi: 10.4067/S0718-07052015000200013.

Solé, Isabel (2011). Competencia lectora y aprendizaje. España, Universidad de Barcelona).

Vega, C. (2012). Niveles de comprensión lectora en alumnos del quinto grado de primaria de una Institución Educativa de Bellavista - Callao". (Tesis de maestría). Universidad san Ignacio de Loyola. Lima, Perú. 